\title{
A Case Report of Lymphocytic Hypophysitis Related to Pregnancy
}

\author{
Estrella Diego1, Andreea Ciudin1, Olga Simo-Servat1, Angel Ortiz1, \\ Liliana Gutierrez-Carrasquilla², Jordi Mesa ${ }^{1}$ \\ ${ }^{1}$ Hospital Universitario Valle de Hebron, Barcelona, Spain \\ ${ }^{2}$ Hospital Arnau de Vilanova, Lleida, Spain \\ Email: e.diego@vhebron.net
}

Received 10 October 2015; accepted 6 December 2015; published 9 December 2015

Copyright (C) 2015 by authors and Scientific Research Publishing Inc.

This work is licensed under the Creative Commons Attribution International License (CC BY).

http://creativecommons.org/licenses/by/4.0/

c) (i)

\section{Open Access}

\section{Abstract}

A 39-year-old woman consulted in the postpartum period because of agalactia, polyuria, polydipsia, and headache and decreased visual acuity. The initial analysis was compatible with a panhypopituitarism. The magnetic resonance (MRI) showed an expansive sellar and suprasellar lesion of high density in the basal study and enhancement after contrast administration, in contact with the optic chiasm, so urgent surgery was indicated. While waiting for the surgery, intravenous corticosteroids were initiated and the symptoms rapidly resolved. Most of the pituitary axes were recovered, except the diabetes insipidus. The control MRI showed complete resolution of the sellar mass. The surgery was canceled and the diagnosis of lymphocyte hypohpyisitis was made. Lymphocyte hypophysitis is an uncommon disease in which inflammation of the pituitary gland occurs. This results in a permanent or transient hormonal insufficiency. Lymphocyte hypophysitis is a very rare condition that occurs preferentially in women in the peripartum period. Early initiation of high doses of systemic corticosteroids may avoid unnecessary surgery in some cases.

\section{Keywords}

Hypophysitis, Pregnancy, Panhypopituitarism, Lymphocytic Hypophysitis

\section{Introduction}

Lymphocytic hypophysitis is a very rare condition in which an inmune inflammation of the pituitary gland occurs. In general, the lymphocytic hypophysitis is seen in women and is associated with the peri- and postpartum period. It is estimated an incidence of one case per nine million people, but probably, there are not diagnosticated subclinical forms [1]. Initially, the histopathology consists of a monoclonal lypmphocityc infiltrate which 
can leave minimal sequelae or progress to fibrosis. This may result in temporary or permanent hormonal dysfunction. The clinical presentation varies depending on the pituitary segment that is affected and the tumor mass. The therapeutic approach is controversial. There are usually large pituitary tumors, associated with locally compressive symptoms, and often the transesphenoidal surgery is performed. Nevertheless, the conservative medical management is justified in many cases, given the self-limited nature of the inflammatory process. Here we describe the case of a large pituitary mass due to lymphocytic hypophysitis, associated with visual loss, in a patient in the postpartum period with an unexpected improvement after starting systemic corticosteroids.

\section{Case Report}

A 39-year-old young woman, natural from Algeria, was referred to our hospital because of headache and impaired vision. As personal history, she gave birth 6 months ago, to a live newborn, in the 38th week of gestation.

The patient reported agalactia after the first week of postpartum; one month later she started with polyuria, polydipsia and nocturia; episodes of dizziness, hypotension and amenorrhea. Three months after childbirth, she suffered from an holocraneal tension-type, headache, with partial improvement with conventional analgesia. Finally, she presented decreased visual acuity so she consulted to the emergency unit.

In the emergency department, the fundus opthalmoscopy resulted normal. The campimetric evaluation showed a significant decrease in visual acuity in the left eye (she only saw the movement of the hands) and reduction in the right eye (2 meters away). The patient was hemodynamically stable, blood pressure was 130/72 $\mathrm{mmHg}$, and the rest of the physical examination had no significant findings (Table 1). The first blood tests were normal (glucose $85 \mathrm{mg} / \mathrm{dL}$ (74 - 110), sodium $137 \mathrm{mmol} / \mathrm{L}$ (136 - 146), potassium $3.49 \mathrm{mmol} / \mathrm{L}$ (3.4 - 4.5), creatinine $0.50 \mathrm{mg} / \mathrm{dL}$ (0.51 - 0.95), hemoglobin $12.8 \mathrm{~g} / \mathrm{L}$ (11.8 - 14.7), leucocytes 12,890/L (4100 - 9900)). The craneal computed axial tomography (CT) showed an expansive sellar and suprasellar lesion of high density in the basal study and enhancement after contrast administration. The size of the lesion was $18 \times 16 \mathrm{~mm}$, and it contacted with the optic chiasm. The image suggested a pituitary adenoma with suprasellar extension or less likely a lymphocyte hypophysitis.

A cranial magnetic resonance (MRI) was performed in order to better characterize the pituitary mass and it confirmed the findings seen on the CT (Figure 1).

The hormonal study was compatible with a panhypopituitarism (thyrotropin $0.14 \mathrm{mU} / \mathrm{L}$ (0.55 - 4.78), free thyroxine $0.73 \mathrm{ng} / \mathrm{dL}(0.80$ - 1.76), corticotropin < $1.6 \mathrm{pg} / \mathrm{mL}$ (4.7 - 48.8), cortisol 0.77 microg/dL (4.2 - 22.4), $\mathrm{LH}<0.12 \mathrm{U} / \mathrm{L}(1.9$ - 12.5), FSH $0.6 \mathrm{U} / \mathrm{L}(2.5$ - 10.2), prolactin $25.94 \mathrm{ng} / \mathrm{mL}(2.8$ - 29.2), estradiol $19.33 \mathrm{pg} / \mathrm{mL}$ (19.5 - 144.2), progesterone $0.22 \mathrm{ng} / \mathrm{mL}$ (3.34 - 25.56), somatotropin $0.18 \mathrm{ng} / \mathrm{mL}(0$ - 8), IGF-1 $29.57 \mathrm{ng} / \mathrm{mL}$ (78 - 274)). Levothyroxine $25 \mathrm{mcg}$ per day was also initiated. Diuresis of $3000 \mathrm{ml}$ in 12 hours and hypernatremia of $159 \mathrm{mmol} / \mathrm{L}$ were recorded. We suspected a insipidus diabetes of central origin, so we initiated desmopressin 10 mgcs every 12 hours oral.

The patient was admitted to the Intensive Care Unit pending surgery and $6 \mathrm{mg}$ of dexamethasone intravenously every 8 hours was started.

\begin{tabular}{lc} 
Table 1. Physical exam. & \\
\hline Gender & Female \\
Age & 39 years old \\
Blood pressure & $130 / 72 \mathrm{mmHg}$ \\
Neurological exam: & \\
Conscience & Alert \\
Campimetry & Decrease in visual acucity \\
Fundus opthalmoscopy & Normal \\
Sensitivity exam & Normal \\
Motor exam & Normal \\
Cardiovascular exam: & Normal \\
\hline
\end{tabular}




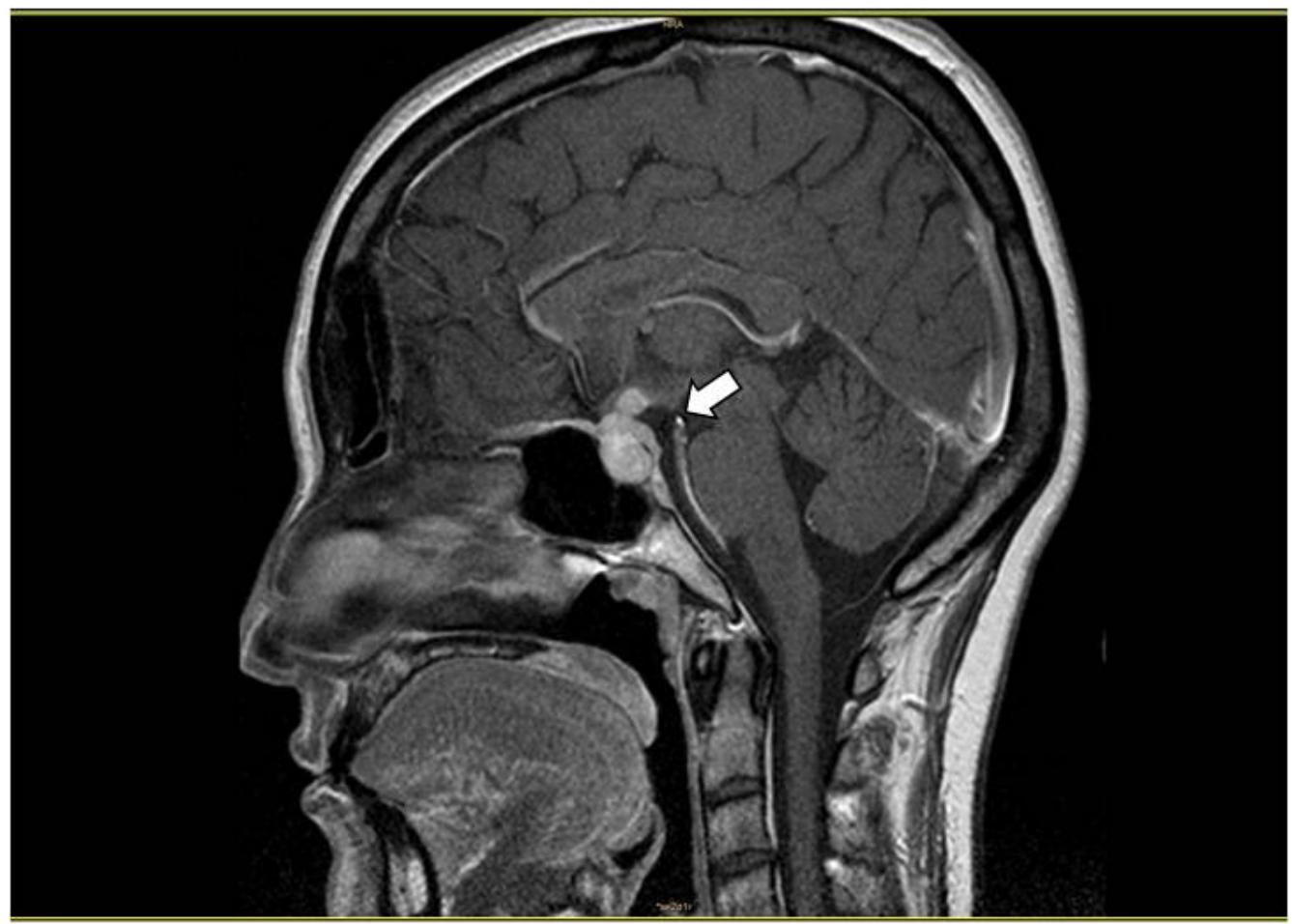

Figure 1. Initial RMI. The first RMI shows an heterogeneous pituitary gland, increased of size, about 18 $\times 12 \times 15 \mathrm{~mm}$ of diameter, with globular aspect. After administration of intravenous contrast, an heterogeneous enhancement of the pituitary gland is observed. The pituitary stalk shows an important thickening and lengthening from its insertion to the tuber cinerum, with marked enhancement after administration of contrast. The pituitary stalk has a maximum diameter of $7 \mathrm{~mm}$ and compresses the optic chiasm. These features sugest a lymphocytic hypophysitis.

After seven hours of the onset of dexamethasone, the patient had a normalization of the visual acuity, so surgical treatment was postponed and lymphocyte hypophysitis was suspected. In the next 7 days, the patient improved significantly, with a complete recovery of visual acuity and subsequent normalization of the hormonal axes (see Table 2), which allowed a progressive reduction of corticosteroid therapy. Moreover levothyroxine was discontinued.

A control pituitary MRI was performed, revealing a total normalization of the size and signal of the infundibular stalk and adenohypophysis, without any evidence of focal lesions (Figure 2) after 12 days of treatment. The corticosteroid treatment was withdrawn after five weeks in descending pattern. The insipidus diabetes persisted and the patient maintained a proper water balance with desmopressin.

\section{Discussion}

Our case highlights the possible relationship between pregnancy and the development of a lymphocyte hypophysitis, and the excellent response of the lymphocyte hypophysitis to steroid treatment. This condition is more common in women than in men and about 55\% of cases occur during pregnancy or postpartum [2], especially in the last month of pregnancy or in the first two months postpartum, as happened in our case.

The most frequent presentation form is headache and impaired visual acuity, as described by Gutenberg et al. [3]. The average age in their group of patients was 36 years, similar to our case and hormone deficiency was found in $94 \%$ of patients.

Upon arrival to the emergency unit, the first suspected diagnosis was pituitary apoplexy, because it is frequent in peripartum period and the symptoms are the same. In our case the MRI and the spectacular response to corticosteroid treatment oriented the diagnostic and helped us avoid an unnecessary surgery.

The first hormone alteration in our patient seemed to be the hypoprolactinaemia, evidenced by the impossibility of breastfeeding. In most of the cases hyperprolactinemia [2] [4] is observed, due the compressive effect. 
Table 2. Hormonal evolution after initiation of corticosteroid therapy.

\begin{tabular}{cccccc}
\hline Hormone & Day 1 & Day 3 & Day 5 & Day 10 & Normal range \\
\hline TSH (mU/L) & 0.14 & 4.34 & 2.86 & 1.55 & $0.55-4.78$ \\
T4 free (ng/dL) & 0.73 & 0.88 & 1.29 & 1.29 & $0.80-1.76$ \\
ACTH (pg/mL) & $<1.6$ & 28.41 & 13.79 & & $4.77-48.8$ \\
Cortisol (microg/dL) & 0.77 & 14.58 & 25.92 & 12.16 & $4.22-22.4$ \\
LH (U/L) & $<0.12$ & 2.94 & 7.75 & 0.31 & $1.9-12.55$ \\
FSH (U/L) & 0.6 & 7.09 & 15.68 & 5.63 & $2.5-10.2$ \\
Prolactine (ng/mL) & 25.94 & 15.86 & 28.34 & 21.65 & $2.8-29.2$ \\
Estradiol pg/mL & 19.33 & $<11.8$ & $<11.8$ & 22.59 & $19.5-144.2$ \\
Progesterone (ng/mL) & 0.22 & 0.37 & 0.46 & 0.36 & $3.34-25.56$ \\
GH (ng/mL) & 0.18 & 0.28 & & & $0-8$ \\
IGF-1 (ng/mL) & 29.57 & 94.26 & & & $78-274$ \\
\hline
\end{tabular}

Abbreviations: TSH: thyrotropin; T4 free: free thyroxine; ACTH: corticotrophin; LH: luteinizing hormone; FSH: follicle stimulating hormone; GH: somatotropin; IGF-1: insulin-like growth factor 1.

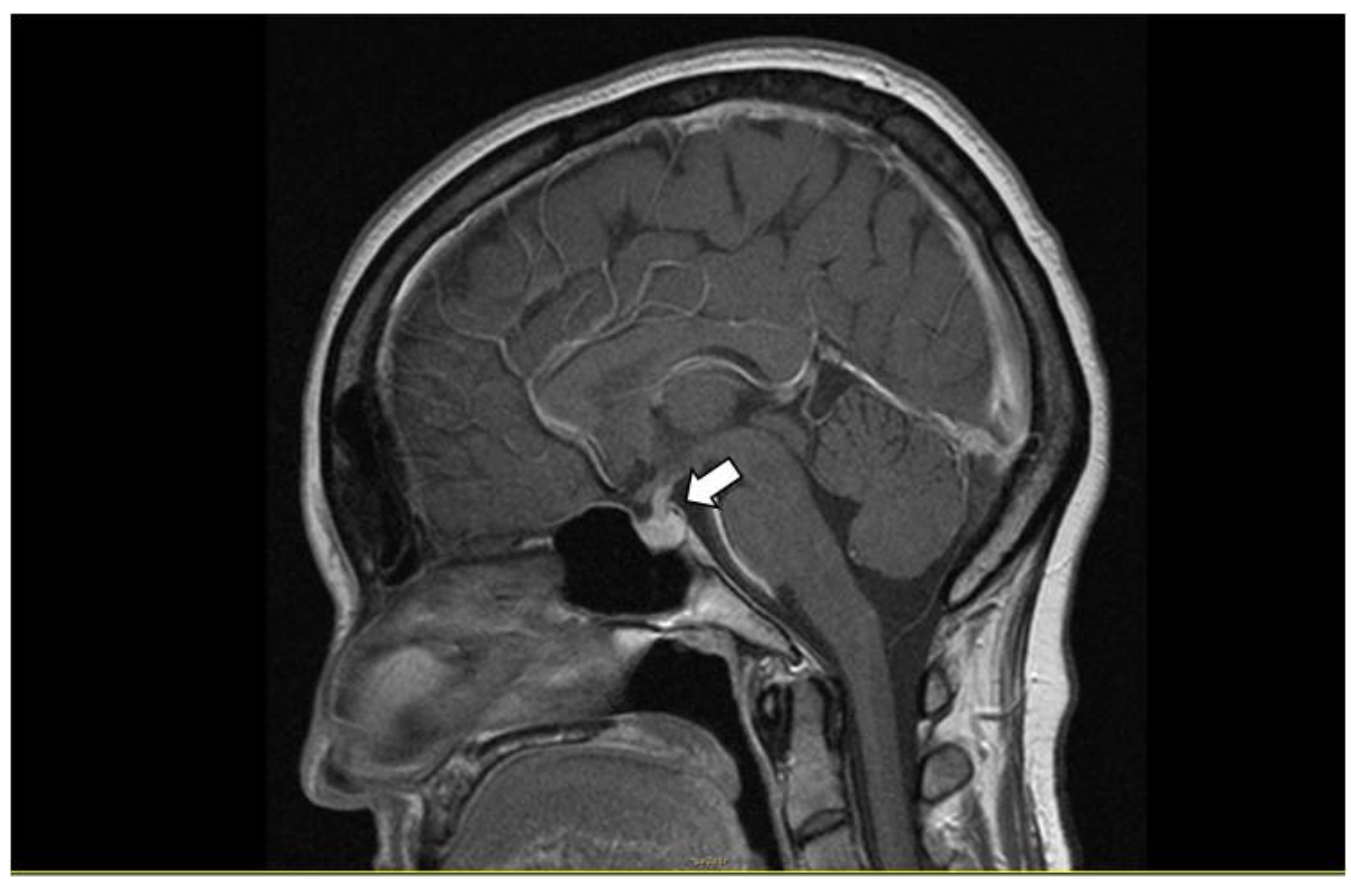

Figure 2. RMI after 12 days of corticosteroids treatment. After 12 days of treatment we can observe normalization of size and signal intensity of the anterior pituitary and the infundibular stalk.

However it is estimated that in up to $11 \%$ of cases inhibition of lactation can be found [1]. The following axis affected was the corticotropin, which is characteristic of hypophysitis. In fact, according to the Luppi's revision this is the most frequently axe affected, seen in about a $42 \%$ - $65 \%$ of all the patients with lymphocytic hypophysitis [4]. Note that this can be a feature to differentiate between a hypophysitis and a pituitary adenoma, in which corticotropin is the last hormone affected (Table 3). Insipidus diabetes unmasks in fund ibuloneurohipofisits, which was observed on MRI, probably caused by autoimmune destruction of the neurohypophysis.

The CT scan only showed an expansive lesion at sellar and suprasellar level, unable to differentiate between 
Table 3. Differences between hypophysitis and pituitary adenoma. Adapted from (6).

\begin{tabular}{ccc}
\hline Characteristics & Hypophysitis & Adenoma \\
\hline Related to pregnancy & Yes & No \\
First axe afected & Adrenal & Somatotropic \\
Volume & $<6 \mathrm{~cm}^{3}$ & $>6 \mathrm{~cm}^{3}$ \\
Gadolinum enhancement & High & Low \\
Symmetry & Symmetric & Asymmetric \\
Posterior pituitary bright & Conserved & Lost \\
Stalk size & Enlarged & Normal \\
\hline
\end{tabular}

an adenoma or hypophysitis. However, MRI is the "gold standard" for the study of hypophysitis. The pituitary gland was enlarged and globular with heterogeneous enhancement after intravenous contrast administration. A significant thickening of the pituitary stalk was observed. According to the series of Imber et al. radiological changes are found in $90 \%$ of patients [5]. The most frequently found radiological sign is the uniform contrast enhancement and the thickening of the pituitary stalk (60\% and $66 \%$ of patients respectively).

Currently there is no radiological finding with sufficient specificity to distinguish a hypophysitis of a pituitary adenoma. Therefore, it has been proposed a radiological score [6] based on the presence of radiological signs and clinical features (related to pregnancy, volume and symmetry of the pituitary mass, signal strength, homogeneity of the signal after administration of gadolinium, brightness presence of the posterior pituitary gland, the pituitary stalk size and thickness of the mucosa). The sum of this items help to differentiate between a hypophysitis or a pituitary adenoma. The score in our case was -8 , which was indicative of hypophysitis.

The inicial reason for starting corticosteroid treatment in our patient was the adrenal insufficiency. The unexpected resolution of headache and visual deficit in less than 12 hours orientated to the diagnostic of lymphocyte hypophysitis. Lupi et al. reviewed 40 patients with hypophysitis treated with glucocorticoids [4]. A reduction in the size of the adenohypophysis was found in $87 \%$ of patients treated with oral corticosteroids, the function of the adenohypophysis improved in $46 \%$ of these patients and the function of the neurohypophysis in $18 \%$. $75 \%$ of patients treated with endovenous glucocorticoids had a reduction in the size of the hypophysis, the adenohypophysis function improved in $54 \%$ of patients and the neurohypophysis function in $66 \%$. In our patient, all the symptoms improved and so did the majority of the hormone deficiency, except diabetes insipidus. We should emphasize that the pituitary stalk was normalized in the MR.

The amelloriation with glucocorticoid therapy manifest the autoinmune etiology of the hypophysitis, and probably other inmunosupressive drugs could be use in these cases. Glucorticosteroids improve compression symptoms and sustitues the adrenal axis. This treatment must be valorated in patients with lymphocyte hypophysitis and probably surgery could be avoided in many cases.

Although we don't dispose of an anatomic pathological exam for an accurate diagnosis, the clinical features, MRI images and the response to corticosteroids, strongly suggests the diagnostic of a lymphocyte hypophysitis. As mentioned previously, about half of the cases of lymphocytic hypophysitis occurred around pregnancy [7]. Several arguments have been exposed for this. Among them, the most accepted theory is the existence of autoantigens that react with both the placenta and the pituitary gland. In 1998 Decrock demonstrated that up to 70\% of patients with biopsy-proven of lymphocyte hypophysitis showed autoantibodies against cytosolic protein 49 $\mathrm{kDa}$ [8]. However, this antibody was also found in patients with other autoimmune diseases or pituitary alterations. O'Dwyer found that another antibody, the neuronal specific enolase (NSE), is expressed in both pituitary and placenta cell [7] [8]. It seems that there is a theoretical basis that explains the predilection of the peripartum period for lymphocyte hypophysitis. However more studies are needed to verify this theory.

\section{Conclusion}

Lymphocyte hypophysitis is a rare disease that occurs preferentially in women in the peripartum period. We should suspect it if we found a patient with a pituitary lesion, compression symptoms and hormone deficiency. In this situation early initiation of high doses of systemic corticosteroids may avoid unnecessary surgery. 


\section{References}

[1] Català Bauset, M., Gilsanz Peral, A., Girbés Borràs, J., Zugasti Murillo, A., Moreno Esteban, B., Halperin Rabinovich, I., Obiols Alfonso, G., Picó Alfonso, A., Del Pozo Picó, C., et al. (2008) Clinical Practice Guideline for the Diagnosis and Treatment of Hypophysitis. Endocrinología y Nutrición, 55, 44-53. http://dx.doi.org/10.1016/S1575-0922(08)70634-X

[2] Rivera, J.A. (2006) Lymphocytic Hypophysitis: Disease Spectrum and Approach to Diagnosis and Therapy. Pituitary, 9, 35-45. http://dx.doi.org/10.1007/s11102-006-6598-z

[3] Gutenberg, A., Hans, V., Puchner, M.J., Kreutzer, J., Brück, W., Caturegli, P. and Buchfelder, M. (2006) Primary Hypophysitis: Clinical-Pathological Correlations. European Journal of Endocrinology, 155, 101-107. http://dx.doi.org/10.1530/eje.1.02183

[4] Lupi, I., Manetti, L., Raffaelli, V., Lombardi, M., Cosottini, M., Iannelli, A., Basolo, F., Proietti, A., Bogazzi, F., Caturegli, P. and Martino, E. (2011) Diagnosis and Treatment of Autoimmune Hypophysitis: A Short Review. Journal of Endocrinological Investigation, 34, e245-e252.

[5] Imber, B.S., Lee, H.S., Kunwar, S., Blevins, L.S. and Aghi, M.K. (2014) Hypophysitis: A Single-Center Case Series. Pituitary, 18, 630-641. http://dx.doi.org/10.1007/s11102-014-0622-5

[6] Gutenberg, A., Larsen, J., Lupi, I., Rohde, V. and Caturegli, P. (2009) A Radiologic Score to Distinguish Autoinmune Hypophysitis from Non Secreting Pituitary Adenoma Preoperatively. AJNR American Journal of Neuroradiology, 30, 1766-1772. http://dx.doi.org/10.3174/ajnr.A1714

[7] O’Dwyer, D.T., Clifton, V., Hall, A., Smith, R., Robinson, P.J. and Crock, P.A. (2002) Pituitary Autoantibodies in Lymphocytic Hypophysitis Target Both Gamma- and Alpha-Enolase-A Link with Pregnancy? Archives of Physiology and Biochemistry, 110, 94-98. http://dx.doi.org/10.1076/apab.110.1.94.897

[8] O’Dwyer, D.T., Smith, A.I., Matthew, M.L., Andronicos, N.M., Ranson, M., Robinson, P.J. and Crock, P.A. (2002) Identification of the 49-kDa Autoantigen with Lympocytic Hypophysitis as Alpha-Enlase. Journal of Clinical Endocrinology \& Metabolism, 87, 752-757. http://dx.doi.org/10.1210/jc.87.2.752 